\title{
Large-scale planetesimal formation by streaming instability
}

\author{
Chao-Chin Yang and Anders Johansen \\ Lund Observatory, Department of Astronomy and Theoretical Physics, Lund University \\ Box 43, SE-22100 Lund, Sweden \\ email: ccyang@astro.lu.se
}

\begin{abstract}
In current theory of planet formation, streaming instability is one of the most promising mechanisms to overcome the meter-barrier in the course of core accretion. Almost all previous works, however, were focused on a local region of protoplanetary disks with a limited size of about 0.2 gas scale heights. Only one radial filamentary particle concentration was seen in these studies. To address this, we conduct the largest-scale simulations of this kind to date, up to 0.8 gas scale heights both horizontally and vertically. We demonstrate that streaming instability remains robust on large scale and multiple radial particle concentrations exist in large enough boxes. This result may be important in characterising the feeding zone of planetesimal formation.
\end{abstract}

Keywords. Hydrodynamics, instabilities, methods: numerical, planetary systems: formation

It has been a long standing problem in core accretion scenario of planet formation for meter/decimetre-sized solid objects to remain in the gaseous protoplanetary disks due to constant head wind (Weidenschilling 1977). One of the most promising mechanisms to overcome this meter-barrier is the streaming instability (Youdin \& Goodman 2005), in which viscous interaction between solids and gas can concentrate the solids to the extent that gravitational collapse takes over to produce Ceres-sized planetesimals (Johansen et al. 2007). Systemic study of this mechanism seems warranted to explore the preferable conditions and the properties of the resulting solid concentration.

We conduct the largest-scale hydrodynamical simulations of this kind to date, up to $0.8 H$ both horizontally and vertically, where $H$ is the gas scale height. We use the Pencil Codet to model a local shearing box of gas and solid particles. The vertical component of the stellar gravity is included so that the particles quickly settle into a thin midplane layer. The gas and particles interact by viscous drag with a Stokes number of 0.3, where the Stokes number is defined as the product of local orbital frequency and particle stopping time due to gas drag. The total solid-to-gas ratio is fixed at 0.02 , which is approximately over the critical solid-to-gas ratio required to trigger strong clumping of these particles (Johansen, Youdin, \& Mac Low 2009).

The top panel of Figure 1a demonstrates the process of particle sedimentation and turbulent stirring. The particle scale height first decreases to a minimum within about one local orbital period and then increases due to stirring by the streaming instability. There exists a significant increase in particle scale height when increasing the vertical size of the simulation box from $0.2 H$ to $0.4 H$, while the results are similar for runs of vertical sizes $0.4 H$ and $0.8 H$. This indicates there exists a minimum vertical size of about $0.4 H$ to properly capture the turbulent stirring of particles by streaming instability.

Furthermore, the concentration of particles by streaming instability seems also dependent on the dimensions of the simulation box, as shown in the bottom panel of Figure 1a.

$\dagger$ The code is publicly available at https://code.google.com/p/pencil-code/. 

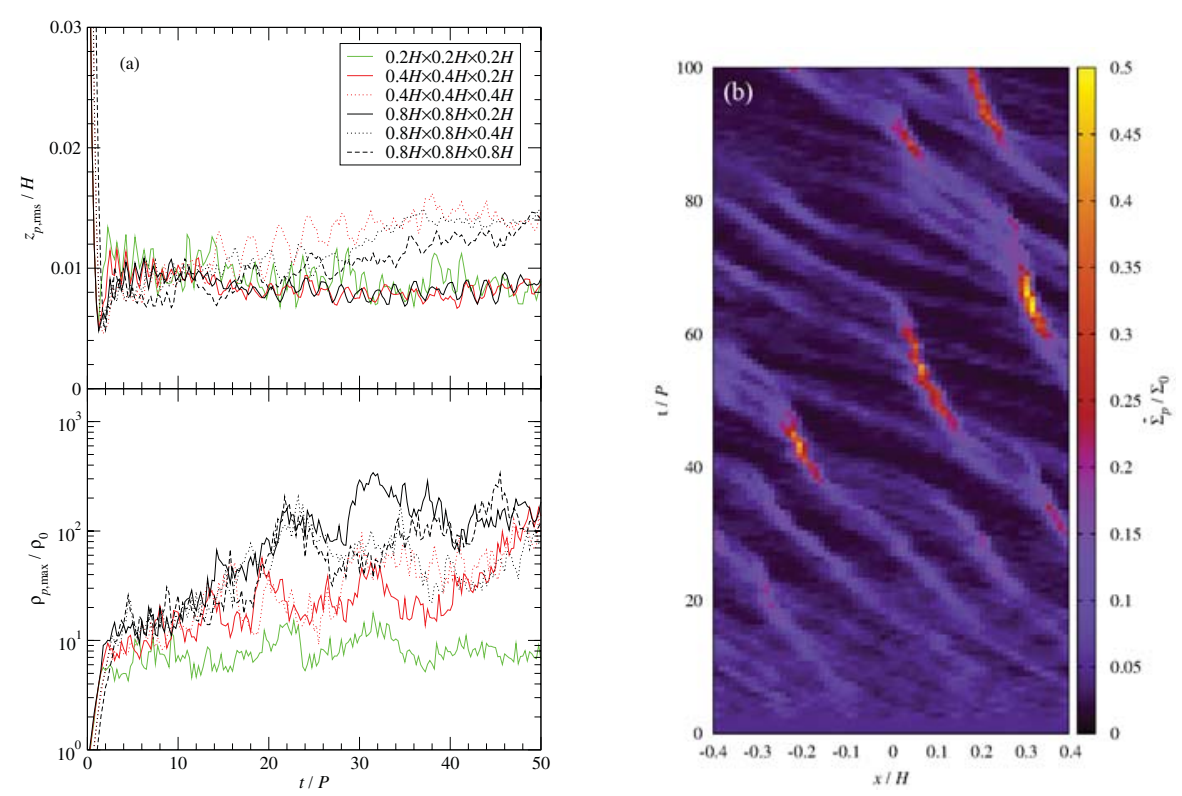

Figure 1. (a) Average particle scale height (top) and maximum particle density (bottom) as a function of time in terms of the local orbital period. Different lines denote simulation boxes of different dimensions, where $H$ is the gas scale height. (b) Space-time diagram of the particle density integrated over the vertical direction and averaged over the azimuthal direction from a $0.8 H \times 0.8 H \times 0.8 H$ simulation box. The horizontal axis represents the radial direction, while the vertical axis denotes the time.

The larger the horizontal dimensions, the higher the peak particle density. On the other hand, the peak particle density seems little affected by the vertical dimension of the simulation box. This phenomenon could be understood by the difference in the size of the particle feeding zone in the azimuthal direction.

Finally, Figure 1b shows the particle density, integrated over the vertical direction and averaged over the azimuthal direction, as a function of time and radial position. In contrast to previous works, where only one radial filamentary structure in particle density was seen, we find multiple concentrations at different radii given large enough horizontal dimension. For a $0.8 H \times 0.8 H \times 0.8 H$ simulation box, at least two particle concentrations exist simultaneously. This indicates that we might have captured the characteristic wavelength of particle concentrations in the non-linear stage of the streaming instability, which helps characterising the feeding zone of planetesimal formation.

In summary, we have systematically investigated the streaming instability of viscously interacting gas and solid particles in a protoplanetary disk with various box dimensions. We find convergence in final particle scale height with vertical dimension. We have also demonstrated multiple radial concentrations of solid particles do exist in large enough simulation boxes, which may have important implications in planet formation scenarios.

\section{References}

Johansen, A., Oishi, J. S., Mac Low, M.-M., et al. 2007, Nature, 448, 1022

Johansen, A., Youdin, A., \& Mac Low, M.-M. 2009, ApJ, 704, L75

Weidenschilling, S. J. 1977, MNRAS, 180, 57

Youdin, A. N. \& Goodman, J. 2005, ApJ, 620, 459 